\title{
A Note on the Numerical Integration of Differential Equations ${ }^{1}$
}

\author{
By W. E. Milne ${ }^{2}$ \\ An integration method for ordinary differential equations is developed, in which the \\ approximation formulae contain derivatives of higher order than those contained in the \\ differential equation itself. The method is particularly useful for linear differential equa- \\ tions. Numerical examples are given for Bessel's' differential equation.
}

\section{Introduction}

The object of this note is to present a method for the numerical integration of ordinary differential equations that appears to possess rather outstanding advantages when applied to certain types of equations. The equations to which the method most readily applies are those for which it is possible to obtain, in comparatively simple form, expressions for two additional derivatives. That is, for an equation of $n$-th order

$$
y^{(n)}=f\left(x, y, y^{\prime}, \ldots, y^{(n-1)}\right),
$$

we obtain by differentiation expressions for $y^{(n+1)}$ and $y^{(n+2)}$. If these expressions are not so involved as to make the labor of substitution prohibitive, then the method here proposed is applicable.

The advantages claimed for the process are:

(1) The start of the integration is accomplished by the same formulas that are used in the regular routine of the process, so that no special formulas or procedures are required in order to get the computation underway.

(2) Each step of the integration makes use of only two lines of the computation, whereas a method employing differences and having a comparable degree of accuracy would require five lines in the computation.

(3) A change in the length of step for step-bystep integration is often necessary as the integra-

1 The preparation of this paper was sponsored (in part) by the Office of Naval Research.

2 Mathematics Department, Oregon State College. tion proceeds. Such a change can be more readily made in this process than where five-line formulas of integration are employed.

(4) The coefficients occurring in the formulas are simpler than those in comparable five-line quadrature formulas, so that the machine calculation is not at all complicated.

The most obvious disadvantage of the process is that it requires the calculation of two additional derivatives at each step, and the labor of substitution in certain instances may be excessive. In such cases this method is not recommended. On the other hand, for equations of simple analytical form, and particularly for linear differential equations, it should prove valuable.

\section{Derivation of Formulas}

Let $x_{0}$ and $x_{1}$, where $x_{1}-x_{0}=h$, be two values of the independent variable $x$, and let $y_{0}, y_{0}^{\prime}$, etc.. $y_{1} y_{1}^{\prime}$ etc., be the corresponding values of $y, y^{\prime}, \ldots$ Assuming that $y$ has a continuous derivative of order 7 we may express $y, y^{\prime}, y^{\prime \prime}$, and $y^{\prime \prime \prime}$ by Taylor's series with remainder term, as follows:

$$
\begin{aligned}
y_{1}= & y_{0}+h y_{0}^{\prime}+\frac{h^{2} y_{0}^{\prime \prime}}{2 !}+\frac{h^{3} y_{0}^{(3)}}{3 !}+\frac{h^{4} y_{0}^{(4)}}{4 !}+\frac{h^{5} y_{0}^{(5)}}{5 !}+\frac{h^{6} y_{0}^{(6)}}{6 !}+ \\
& R_{1}, \\
h y_{1}^{\prime}= & h y_{0}^{\prime}+h^{2} y_{0}^{\prime \prime}+\frac{h^{3} y_{0}^{(3)}}{2 !}+\frac{h^{4} y_{0}^{(4)}}{3 !}+\frac{h^{5} y_{0}^{(5)}}{4 !}+\frac{h^{6} y_{0}^{(6)}}{5 !}+R_{2}
\end{aligned}
$$

$h^{2} y_{1}^{\prime \prime}=h^{2} y_{0}^{\prime \prime}+h^{3} y_{0}^{(3)}+\frac{h^{4} y_{0}^{(4)}}{2 !}+\frac{h^{5} y_{0}^{(5)}}{3 !}+\frac{h^{6} y_{0}^{(6)}}{4 !}+R_{3}$ 


$$
\begin{aligned}
h^{3} y_{1}^{\prime \prime \prime}= & h^{3} y_{0}^{(3)}+h^{4} y_{0}^{(4)}+\frac{h^{5} y_{0}^{(5)}}{2 !}+\frac{h^{6} y_{0}^{(6)}}{3 !}+R_{4}, \\
y_{2}= & y_{0}+2 h y_{0}^{\prime}+\frac{4 h^{2} y_{0}^{\prime \prime}}{2 !}+\frac{8 h^{3} y_{0}^{(3)}}{3 !}+\frac{16 h^{4} y_{0}^{(4)}}{4 !}+ \\
& \frac{32 h^{5} y_{0}^{(5)}}{5 !}+\frac{64 h^{6} y_{0}^{(6)}}{6 !}+R_{5} .
\end{aligned}
$$

From the four equations (1, 2, 3, and 4), the three quantities $y_{0}^{(4)}, y_{0}^{(5)}$, and $y_{0}^{(6)}$ may be eliminated, and the resulting equation can be rearranged so as to give

$y_{1}-y_{0}=\frac{h}{2}\left(y_{1}^{\prime}+y_{0}^{\prime}\right)-\frac{h^{2}}{10}\left(y_{1}^{\prime \prime}-y_{0}^{\prime \prime}\right)+\frac{h^{3}}{120}\left(y_{1}^{\prime \prime \prime}+y_{0}^{\prime \prime \prime}\right)+$ $R_{6}$.

It may be shown by a separate investigation ${ }^{3}$ that

$$
R_{6}=\frac{-h^{7} y^{(7)}(s)}{100,800}
$$

in which $x_{0}<s<x_{1}$.

In a similar manner from eq 1 to 5 we may derive

$$
\begin{gathered}
y_{2}-2 y_{1}+y_{0}=7 h\left(y_{1}^{\prime}-y_{0}^{\prime}\right)-3 h^{2}\left(y_{1}^{\prime \prime}+y_{0}^{\prime \prime}\right)+ \\
\frac{h^{3}}{12}\left(11 y_{1}^{\prime \prime \prime}-5 y_{0}^{\prime \prime \prime}\right)+\frac{210 h^{7} y^{(7)}(s)}{100,800}
\end{gathered}
$$

with $s$ in the interval $x_{0}<s<x_{2}$. These are the required formulas.

\section{Application to Equations of First Order}

Let the given differential equation be

$$
y^{\prime}=f(x, y) \text {. }
$$

Differentiation gives

and

$$
y^{\prime \prime}=f_{x}(x, y)+f_{y}(x, y) y^{\prime},
$$

$y^{\prime \prime \prime}=f_{x x}(x, y)+2 f_{x y}(x, y) y^{\prime}+f_{y y}(x, y) y^{\prime 2}+f_{y}(x, y) y^{\prime \prime}$.

Let the given initial values be $x_{0}, y_{0}$. Then from eq 8,9 , and 10 in succession we obtain $y_{0}^{\prime}, y_{0}^{\prime \prime}, y_{0}^{\prime \prime \prime}$, giving the first line of the computation

${ }^{3}$ W. E. Milne, The remainder in linear methods of approximation, J. Research NBS 43, 501 (1949) RP2042.
To proceed we assume a trial value for $y_{1}$. fairly good trial value is provided by

$$
y_{1}=y_{0}+h y_{0}^{\prime}+\frac{h^{2} y_{0}}{2 !}+\frac{h^{3} y_{0}^{\prime \prime \prime}}{3 !} \text {. }
$$

Next with $x_{1}$, and the trial value $y_{1}$, we obtain trial values for $y_{1}^{\prime}, y_{1}^{\prime \prime}, y_{1}^{\prime \prime \prime}$ from eq 8,9 , and 10 and have the trial line for $x_{1}$ :

$$
\begin{array}{lllll}
x_{1} & y_{1} & y_{1}^{\prime} & y_{1}^{\prime \prime} & y_{1}^{\prime \prime \prime} .
\end{array}
$$

Now using eq 6 we secure an improved value of $y_{1}$, compute $y_{1}^{\prime}, y_{1}^{\prime \prime}, y_{1}^{\prime \prime \prime}$, from eq 8,9 , and 10 , recalculate $y_{1}$ by 2 , (eq 6 ), and repeat this sequence of steps until no change occurs in the value obtained for $y_{1}$. This is taken as correct, and we have two lines of the computation:

$$
\begin{array}{lllll}
x_{0} & y_{0} & y_{0}^{\prime} & y_{0}^{\prime \prime} & y_{0}^{\prime \prime \prime}, \\
x_{1} & y_{1} & y_{1}^{\prime} & y_{1}^{\prime \prime} & y_{1}^{\prime \prime \prime} .
\end{array}
$$

We are now in a position to use formula 7 in order to calculate a trial value for $y_{2}$. Then trial values of $y_{2}^{\prime}, y_{2}^{\prime \prime}, y_{2}^{\prime \prime \prime}$ are obtained from eq 8,9 , and 10 , and we leave the trial line:

$$
\begin{array}{lllll}
x_{2} & y_{2} & y_{2}^{\prime} & y_{2}^{\prime \prime} & y_{2}^{\prime \prime \prime} .
\end{array}
$$

Formula 6 (with subscripts advanced by 1 ) gives an improved value for $y_{2}$. If the "improved" value of $y_{2}$ is different from the "trial" value of $y_{2}$, it will be necessary to recompute $y_{2}^{\prime}, y_{2}^{\prime \prime}, y_{2}^{\prime \prime \prime}$ and apply eq 6 again, repeating these steps until no further change occurs. At this stage we have completed three lines of the computation:

$\begin{array}{lllll}x_{0} & y_{0} & y_{0}^{\prime} & y_{0}^{\prime \prime} & y_{0}^{\prime \prime \prime}, \\ x_{1} & y_{1} & y_{1}^{\prime} & ?_{1}^{\prime \prime} & y_{1}^{\prime \prime \prime}, \\ x_{2} & y_{2} & y_{2}^{\prime} & y_{2}^{\prime \prime} & y_{2}^{\prime \prime \prime} .\end{array}$

Subsequent lines of the calculation are obtained by exactly the same steps as were used to get $y_{2}$.

\section{Discussion of the Process}

This completes the description of the process for the case of equations of the first order. Some comments of a practical nature are, however, pertinent.

(1) Obviously, the error in $y$ at any step due to the use of the approximate formula 6 is bounded by the quantity

$$
E=\frac{h^{7} M}{100,800},
$$


in which $M=\max \left|y^{(7)}\right|$ in the interval covered by the step. Supposing for the moment that $M$ is a known constant, we see that the above equation connects the magnitude of the error $E$ with the length of the step-interval $h$.

If, for example, the problem in question requires that $y$ be obtained accurately to a specified number of decimal places, eq 11 enables us to select an appropriate value of $h$ that will secure this accuracy. On the other hand, if we have already decided on the value of $h$, eq 11 will tell us how many decimals in the result may be regarded as correct.

(2) Actually, $M$ is rarely constant from step to step, and moreover the value of $M$ is unknown since it is ordinarily utterly impractical to calculate the value of the seventh derivative of $y$ at each step. However, a crude estimate (which actually proves to be sufficiently satisfactory) may be made as follows: Assume that the calculation has been performed correctly to the $n$th step so that we have the correct values of the line

$$
\begin{array}{lllll}
x_{n} & y_{n} & y_{n}^{\prime} & y_{n}^{\prime \prime} & y_{n}^{\prime \prime \prime},
\end{array}
$$

a trial value $\bar{y}_{n+1}$ is obtained by eq 7. A final value of $y_{n+1}$ is obtained by eq 6 , repeated if necessary. Now the error of $\bar{y}_{n+1}$ is

$$
\frac{210 h^{7} y^{(7)}(s)}{100,800}
$$

whereas that of $y_{n+1}$ is

$$
-\frac{h^{7} y^{(7)}\left(s^{\prime}\right)}{100,800}+e,
$$

$e$ being the error produced in $y_{n+1}$ by the errors in $y_{n+1}^{\prime}, y_{n+1}^{\prime \prime}, y_{n+1}^{\prime \prime \prime}$, these latter being due to the error of $y_{n+1}$. In actual practice, if the process is rapidly enough convergent for practical use, the error $e$ must be much smaller than the error in $\bar{y}_{n+1}$. Hence, we may neglect $e$. Then

$$
\bar{y}_{n+1}-y_{n+1}=\frac{210 h^{7} y^{(7)}(s)}{100,800}+\frac{h^{7} y^{(7)}\left(s^{\prime}\right)}{100,800} \text {. }
$$

If we ignore the fact that $y^{(7)}(s)$ and $y^{(7)}\left(s^{\prime}\right)$ are not exactly the same, we may add the terms on the right, divide by 211 , and obtain

$$
\text { Error of } y_{n+1}=-\frac{h^{7} y^{(7)}(s)}{100,800}=\frac{y_{n+1}-\bar{y}_{n+1}}{211} \text {. }
$$

Although the foregoing reasoning seems very crude, the final formula

$$
\text { Error of } y_{n+1}=\frac{y_{n+1}-\bar{y}_{n+1}}{211} \text {, }
$$

proves to be not only simple in application but actually quite reliable in determining how many significant figures of the result can be trusted.

(3). At each step of the computation the quantity $c_{n}=y_{n}-\bar{y}_{n}$ should be recorded in a separate column. This column of $c$ 's is used for several purposes.

(a) As long as the $c$ 's vary regularly and have significant figures only in the last two places retained, we proceed with the computation in reasonable confidence that all is well.

(b) A sudden fluctuation in the $c$ 's suggests that a computational mistake has been made, and the lines involved should be rechecked.

(c) If the $c$ 's increase to the point of affecting the last three places retained, then either the interval, $h$, should be shortened, or one less place should be retained.

(d) The necessity for recomputing $y_{n+1}^{\prime}, y_{n+1}^{\prime \prime}$, $y_{n+1}^{\prime \prime \prime}$ can frequently be obviated by estimating $c_{n+1}$ from the known values $c_{n-2}, c_{n-1}, c_{n}$ and adding it to the trial value $\bar{y}_{n+1}$ before computing $y_{n+1}^{\prime}, y_{n+1}^{\prime \prime}, y_{n+1}^{\prime \prime}$. If the estimate is sufficiently accurate, no recomputation is required.

(4). The foregoing discussion applies only after the computation is under way and does not give any clue to the accuracy of $y_{1}$. We would, of course, like to decide on the value of $h$ and on the number of decimal places before starting the computation. This requires that we calculate $y_{0}^{(7)}$ from the differential equation and the initial values.

\section{Equations of Higher Order}

The modifications required to apply the process to equations of second or higher order are slight. In the case of an equation of second order, for example, the routine (after the start has been made) is as follows:

Predict $y_{2}^{\prime}$ by eq 7 modified as follows:

$$
\begin{aligned}
& y_{2}^{\prime}-2 y_{1}^{\prime}+y_{0}^{\prime}=7 h\left(y_{1}^{\prime \prime}-y_{0}^{\prime \prime}\right)-3 h^{2}\left(y_{1}^{\prime \prime \prime}+y_{0}^{\prime \prime \prime}\right)+ \\
& \frac{h^{3}}{12}\left(11 y_{1}^{(4)}-5 y_{0}^{(4)}\right) .
\end{aligned}
$$


Predict $y_{2}$ by eq 7 . Calculate $y_{2}^{\prime \prime}, y_{2}^{\prime \prime \prime}, y_{2}^{(4)}$ from the differential equations and the equations obtained by differentiation.

Correct $y_{2}^{\prime}$ by

$y_{2}^{\prime}-y_{1}^{\prime}=\frac{h}{2}\left(y_{2}^{\prime \prime}+y_{1}^{\prime \prime}\right)-\frac{h^{2}}{10}\left(y_{2}^{\prime \prime \prime}-y_{1}^{\prime \prime \prime}\right)+\frac{h^{3}}{130}\left(y_{2}^{(4)}+y_{1}^{(4)}\right)$.

Correct $y_{2}$ by

$y_{2}-y_{1}=\frac{h}{2}\left(y_{2}^{\prime}+y_{1}^{\prime}\right)-\frac{h^{2}}{10}\left(y_{2}^{\prime \prime}-y_{1}^{\prime \prime}\right)+\frac{h^{3}}{130}\left(y_{2}^{\prime \prime \prime}+y_{1}^{\prime \prime \prime}\right)$.

\section{Illustrative Examples}

The foregoing method is applied to the second order differential equation

$$
x y^{\prime \prime}+y^{\prime}+x y=0,
$$

with conditions $y=1, y^{\prime}=0$ at $x=0$.

Example 1, below, gives the solution by another method using $h=0.1$. Example 2 uses the present method with $h=0.5$. A comparison of the computation time is given. Example 3 uses the present method with $h=0.1$.

It appears that in example 2 the error of $y$ is about 2 in sixth place, whereas in example 3 the error is occasionally 1 in the tenth place.

A comparison of example 1 and example 2 shows that the new method obtained the value of $J_{0}(3)$ in only six steps (and more accurately) than the simple method based on Simpson's Rule could secure in 30 steps. Although the labor of substitution per step is much greater for the new method, the reduction in number of required steps more than offsets this extra work, as is shown by a comparison of required times.

Example 3 provides further evidence of the power of the new method. Anyone with experience in numerical solution of differential equations will recognize that to solve Bessel's equation to 10 decimal places in the neighborhood of the orign with step-intervals of length 0.1 requires a pretty accurate method of solution. 
Differential equation: $x \frac{d^{2} y}{d x^{2}}+\frac{d y}{d x}+x y=0$

Computation formulas

Predictor: $y_{n+1}^{\prime}=y_{n-3}^{\prime}+h\left[4 y_{n-1}^{\prime \prime}+8 / 3 \delta^{2} y_{n-1}^{\prime \prime}\right]$.

Corrector: $y_{n+1}^{\prime}=y_{n-1}^{\prime}+h\left[2 y_{n}^{\prime \prime}+1 / 3 \delta^{2} y_{n}^{\prime \prime}\right]$.

Derivatives: $y^{\prime \prime}=-\frac{1}{x} y^{\prime}-y$.

Time: $2 \frac{1}{4} \mathrm{hr} ; h=0.1$

\begin{tabular}{|c|c|c|c|c|c|c|c|c|c|}
\hline \multirow{2}{*}{$x$} & \multirow{2}{*}{$y$} & \multirow{2}{*}{$y^{\prime}$} & \multirow{2}{*}{$y^{\prime \prime}$} & \multirow{2}{*}{$1 / 3 \delta^{2} y^{\prime}$} & \multirow{2}{*}{$1 / 3 \delta^{2} y^{\prime \prime}$} & \multirow{2}{*}{$c$} & \multirow{2}{*}{$c^{\prime}$} & \multicolumn{2}{|c|}{ True values } \\
\hline & & & & & & & & $y$ & $y^{\prime}$ \\
\hline 0.2 & a0. 990025 & $\mathrm{a}-0.099501$ & -0.492520 & -..... & - & ... & ... & & \\
\hline .3 & ะ. 977626 & a- 148319 & -.483229 & 0.000370 & 0.001203 & ... & $\ldots$ & & \\
\hline .4 & ง. 960398 & $a-.196027$ & -.470330 & .000489 & .001166 & -.. & $\ldots$ & . n & $\ldots$ \\
\hline .5 & в. 938470 & a- .242268 & -.453934 & .000603 & .001122 & ... & $\ldots$ & - n. & - \\
\hline .6 & .912005 & $-.286702(0)$ & -.434168 & .000714 & .001065 & 0 & 2 & 0.912005 & -0.286701 \\
\hline .7 & .881201 & -.328995 & -.411208 & .000815 & .001004 & 0 & 0 & .881201 & -.328996 \\
\hline .8 & .846288 & $-.368843 \quad(2)$ & -.385234 & .000914 & .000930 & 0 & 1 & .846287 & -.368842 \\
\hline .9 & .807524 & -.405949 & -.356470 & .001001 & .000853 & 0 & 1 & .807524 & -.405950 \\
\hline 1. 0 & .765198 & -.440052 & -.325146 & .001085 & .000764 & 0 & 0 & .765198 & -.440051 \\
\hline 1.1 & .719622 & $-.470902(1)$ & -.291529 & .001154 & .000674 & 0 & 1 & .719622 & -.470902 \\
\hline 1. 2 & b. 671133 & -.498290 & -.255891 & .001219 & .000574 & 2 & 0 & .671133 & -.498289 \\
\hline 1.3 & .620086 & $-.522023(1)$ & -.218530 & .001269 & .000473 & 0 & 2 & .620086 & -.522023 \\
\hline 1.4 & $.566855(7)$ & -.541949 & -.179749 & .001313 & .000366 & 2 & 0 & .566855 & -.541948 \\
\hline 1.5 & .511828 & -.557936 & -.139871 & .001342 & .000259 & 0 & 0 & .511828 & -.557937 \\
\hline 1.6 & $.455402(4)$ & -.569897 & -.099216 & .001364 & .000146 & 2 & 0 & .455402 & -.569896 \\
\hline 1.7 & .397985 & -.577765 & -.058123 & .001372 & +.000037 & 0 & 1 & .397985 & -.577765 \\
\hline 1.8 & $.339986(7)$ & -.581518 & -.016920 & .001371 & -.000077 & 1 & -1 & .339986 & -.581517 \\
\hline 1.9 & $.281819(20)$ & $-.581157(6)$ & +.024053 & .001357 & -.000184 & 1 & 1 & .281819 & -.581157 \\
\hline 2. 0 & .223890 & -.576726 & .064473 & .001334 & -.000295 & 2 & -1 & .223891 & -.576725 \\
\hline 2.1 & $.166607(8)$ & $-.568292(1)$ & .104008 & .001298 & -.000398 & 1 & +1 & .166607 & -.568292 \\
\hline 2.2 & .110361 & $-.555964(5)$ & .142350 & .001255 & -.000502 & 2 & -1 & .110362 & -.555963 \\
\hline 2.3 & .055540 & -.539872 & .179187 & .001198 & -.000595 & 1 & 0 & .055540 & -.539873 \\
\hline 2.4 & $.002506(8)$ & $-.520186(8)$ & .214238 & .001136 & -.000689 & 2 & -2 & +.002508 & -.520185 \\
\hline 2.5 & -.048384 & -.497093 & .247221 & .001060 & -.000771 & +1 & 0 & -.048384 & -.497094 \\
\hline 2. 6 & -.096807 & $-.470819(20)$ & .277891 & .000982 & -.000852 & +2 & -1 & -.096805 & -.470818 \\
\hline 2. 7 & $-.142450(49)$ & -.441600 & .306006 & .000890 & -.000919 & +1 & 0 . & -.142449 & -.441601 \\
\hline 2.8 & $-.185038(6)$ & -.409710 & .331363 & .000798 & -.000984 & +2 & -1 & -.185036 & -.409709 \\
\hline 2.9 & -.224312 & -.375426 & .353769 & .000694 & -.001034 & 0 & 0 & -.224312 & -.375427 \\
\hline 3.0 & -.260054 & -.339060 & .373074 & - & - & +1 & -1 & -.260052 & -.339059 \\
\hline
\end{tabular}

a Starting values given.

b In the column for $y$ and $y^{\prime}$ appear the corrected values. The digits of the predicted values when different from the corrected are shown in parentheses. 
Differential equation: $x \frac{d^{2} y}{d x^{2}}+\frac{d y}{d x}+x y=0$

Computation formulas

Predictor: $\quad y_{n+1}^{\prime}=2 y_{n}^{\prime}-y_{n-1}^{\prime}+7 h\left(y_{n}^{\prime \prime}-y_{n-1}^{\prime \prime}\right)-3 h^{2}\left(y_{n}^{\prime \prime \prime}+y_{n-1}^{\prime \prime \prime}\right)+\frac{h^{3}}{12}\left(11 y_{n}^{\mathrm{IV}}-5 y_{n-1}^{\mathrm{IV}}\right)$

$$
y_{n+1}=2 y_{n}-y_{n-1}+7 h\left(y_{n}^{\prime}-y_{n-1}^{\prime}\right)-3 h^{2}\left(y_{n}^{\prime \prime}+y_{n-1}^{\prime \prime}\right)+\frac{h^{3}}{12}\left(11 y_{n}^{\prime \prime \prime}-5 y_{n-1}^{\prime \prime \prime}\right)
$$

Corrector: $\quad y_{n+1}^{\prime}=y_{n}^{\prime}+\frac{h}{2}\left(y_{n+1}^{\prime \prime}+y_{n}^{\prime \prime}\right)-\frac{h^{2}}{10}\left(y_{n+1}^{\prime \prime \prime}-y_{n}^{\prime \prime \prime}\right)+\frac{h^{3}}{120}\left(y_{n+1}^{\mathrm{IV}}+y_{n}^{\mathrm{IV}}\right)$

$$
y_{n+1}=y_{n}+\frac{h}{2}\left(y_{n+1}^{\prime}+y_{n}^{\prime}\right)-\frac{h^{2}}{10}\left(y_{n+1}^{\prime \prime}-y_{n}^{\prime \prime}\right)+\frac{h^{3}}{120}\left(y_{n+1}^{\prime \prime \prime}+y_{n}^{\prime \prime \prime}\right)
$$

Derivatives: $y^{\prime \prime}=\frac{1}{x} y^{\prime}-y$

$$
\begin{aligned}
& y^{\prime \prime \prime}=\frac{2}{x} y^{\prime \prime}-y^{\prime}-\frac{1}{x} y \\
& y^{\mathrm{IV}}=-\frac{3}{x} y^{\prime \prime \prime}-y^{\prime \prime}-\frac{2}{x} y^{\prime}
\end{aligned}
$$

\begin{tabular}{|c|c|c|c|c|c|c|c|c|c|}
\hline \multirow{2}{*}{$x$} & \multirow{2}{*}{$y$} & \multirow{2}{*}{$y^{\prime}$} & \multirow{2}{*}{$y^{\prime \prime}$} & \multirow{2}{*}{$y^{\prime \prime \prime}$} & \multirow{2}{*}{$y^{\mathrm{IV}}$} & \multirow{2}{*}{$c$} & \multirow{2}{*}{$c^{\prime}$} & \multicolumn{2}{|c|}{ True values } \\
\hline & & & & & & & & $y$ & $y^{\prime}$ \\
\hline 0 & a1. & $a_{0}$ & -0.5 & 0 & 0.375 & ... & -.. & $\ldots$ & -... \\
\hline 0.5 & 0.938470 & a -0.242268 & -.453934 & 0.181064 & .336622 & -.- & -.. & - & - n \\
\hline 1.0 & b. $.765195(9)$ & $-.440047(63)$ & -.325148 & .325148 & .229798 & 4 & -16 & 0. 765198 & -0.440051 \\
\hline 1.5 & $.511826(31)$ & $-.557934(5)$ & -.139870 & .403210 & .077382 & 5 & -1 & .511828 & -.557937 \\
\hline 2. 0 & $.223889(2)$ & $-.576721(18)$ & +.064472 & .400304 & -.088207 & -7 & +3 & .223891 & -.576725 \\
\hline 2.5 & $-.048382(6)$ & $-.497090(1)$ & .247218 & .318668 & -.231948 & -4 & -6 & -.048384 & -.497094 \\
\hline 3.0 & $-.260053(47)$ & $-.339057(48)$ & .373066 & .177021 & -.324043 & 6 & +3 & -.260052 & -.339059 \\
\hline
\end{tabular}

Time: $11 \frac{1}{\mathrm{hr}} ; h=0.5$

a Starting values given.

b In the column for $y$ and $y^{\prime}$ appear the corrected values. The digits of the predicted values when different from the corrected are shown in parentheses.

Differential equation: $x \frac{d^{2} y}{d x^{2}}+\frac{d y}{d x}+x y=0$.

EXAMPLE 3.

Computation formulas: (Same as Example 2.).

Computation, $h=0.1$.

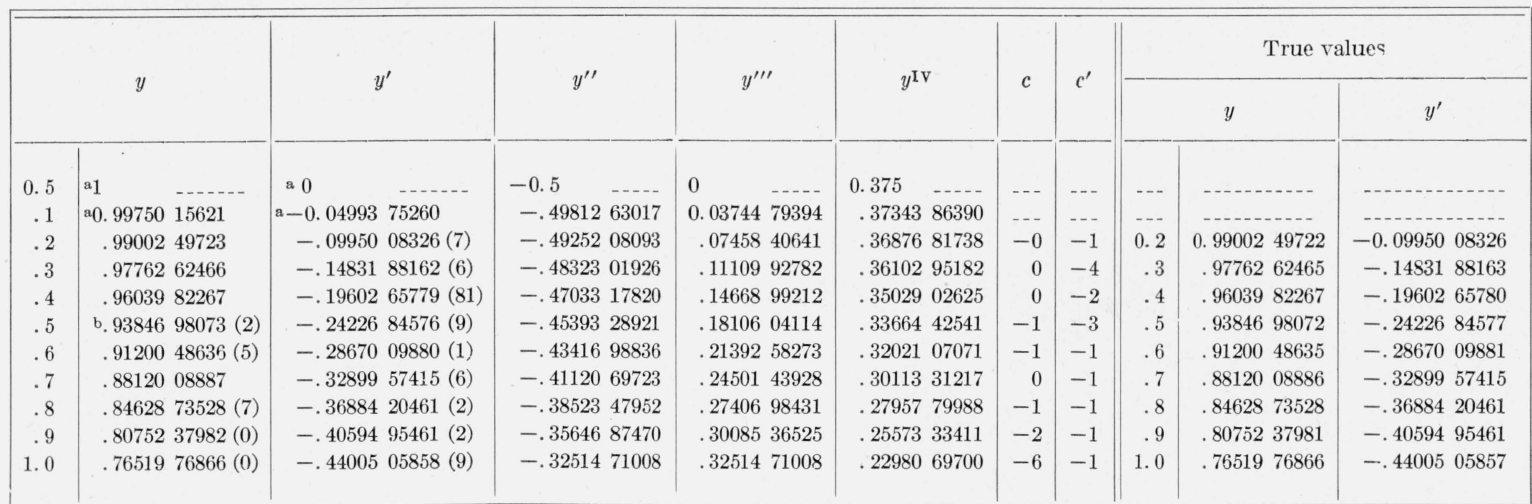

a Starting values given.

b In the column for $y$ and $y^{\prime}$ appear the corrected values. The digits of the predicted values when different from the corrected are shown in parentheses.

Los Angeles, June 17, 1949. 